\title{
EXACT INTEGRATION OF DIFFUSION ORIENTATION DISTRIBUTION FUNCTIONS FOR GRAPH-BASED DIFFUSION MRI ANALYSIS
}

\author{
Brian G. Booth and Ghassan Hamarneh \\ Medical Image Analysis Lab, School of Computing Science \\ Simon Fraser University \\ \{bgb2, hamarneh\}@cs.sfu.ca
}

\begin{abstract}
Graph-based image analysis methods are increasingly being applied to diffusion MRI (dMRI) analysis. Unfortunately, weighting the graph for these methods involves solving a complex integral of an orientation distribution function (ODF). To date, these integrals have been approximated numerically at high computational cost and have resulted in numerical approximation errors that degrade dMRI analysis results. By exploiting a spherical harmonic representation of the ODF, we derive for the first time an analytical solution to the edge weight integrals used in graph-based dMRI analysis. We further show that the computational efficiency of our analytical integration is over forty times faster than numerical approximation schemes on typical data sets. Further, we incorporate our exact integration scheme into an existing graph-based probabilistic tractography method and show a reduction in error accumulation in the resulting tractograms.
\end{abstract}

Index Terms - Diffusion MRI, graph-based analysis, tractography, numerical analysis, spherical harmonics

\section{INTRODUCTION}

Graph-based image analysis methods are popular due to their short computation time, guarantees on optimality and error bounds, and their ability to easily encode spatial relationships (e.g., via a Markov random field assumption) [1]. These graph-based methods are being increasingly used to perform diffusion MRI (dMRI) analysis, tractography in particular [2, $3,4]$. A key aspect of applying graph-based methods to the problem of tractography is to obtain a weighting of the graph that effectively captures the diffusion information.

In an image analysis context, a graph $G=(V, E)$ typically consists of a set of nodes that represent the voxels of a diffusion MR image $I$, i.e., $V=\{v \in \Omega\}$, where $\Omega$ is the discretized domain of $I$. The edges $e_{i j} \in E, E=V \times V$, encode spatial relationships between image voxels $i$ and $j$ (e.g., 26connectivity). Finally, a weight function $w\left(e_{i j}\right): E \rightarrow \mathbb{R}^{+}$ assigns a strength to each edge. For graph-based tractography methods, a widely used weighting function assigns an edge $e_{i j}$ a strength that is proportional to the probability of an anatomical connection between voxels $i$ and $j[2,3,4]$ :

$$
w\left(e_{i j}\right) \propto P_{o d f}\left(i, \vec{r}_{i j}\right)+P_{o d f}\left(j, \vec{r}_{j i}\right)
$$

where $\vec{r}_{i j}$ is the direction of the vector connecting the voxels implied by edge $e_{i j}$ and $P_{\text {odf }}$ is given by

$$
P_{o d f}\left(v, \vec{r}_{u v}\right)=\frac{1}{Z(v)} \int_{(\theta, \phi) \in \beta} \psi_{v}(\theta, \phi) d S
$$

Here, $\beta$ is the solid angle around the edge direction $\vec{r}_{u v}$ and $\psi_{v}$ is the fiber (or sharpened diffusion) orientation distribution function (ODF) at voxel $v$. The variable of integration $d S$ is the infinitesimal spherical surface element and $Z(v)$ is a scaling constant set as in $[2,4]$. Typically, $\beta$ spans the spherical cap of a cone centered around $\vec{r}_{u v}[2,4]$.

The integration in (2) has been shown to be necessary in capturing the diffusion information between voxels, particularly in areas of high diffusion anisotropy where the direction of maximal diffusion does not align with an edge [3]. To date, this ODF integral has been approximated numerically using a tessellation of the sphere [2, 3, 4]. This numerical integration is a concern not only in terms of approximation error, but also in terms of the computational burden of performing numerical integration for the large number of edges connecting voxels in a typical 3D dMRI field.

In this paper, we derive an exact analytical solution to the edge weight integration in (2). Like other analytical solutions in the area of dMRI $[5,6]$, ours ensures numerical accuracy while offering over a fortyfold reduction in computation time. We show how the derivation of this analytical solution is achieved using a spherical harmonic representation of the ODF along with precise angular rotations that address the complicated limits of integration. Further, we apply our solution to the tractography method in [4] and show decrease in error of over fifteen percent. 


\section{THE ODF IN SPHERICAL HARMONICS}

Spherical harmonics have been a popular choice in representing dMRI data modeled in various forms, including diffusion tensors [7], fiber ODFs [8], and diffusion ODFs [9]. An ODF $\psi_{v}(\theta, \phi)$ at voxel $v$ can be represented using a real spherical harmonic expansion as,

$$
\psi_{v}(\theta, \phi)=\sum_{\ell=0}^{K} \sum_{m=-\ell}^{\ell} F_{\ell}^{m} Y_{\ell}^{m}(\theta, \phi)
$$

where $F_{\ell}^{m}$ are coefficients and $Y_{\ell}^{m}(\theta, \phi)$ are the harmonic basis functions. The integer $m:(-\ell \leq m \leq \ell)$ is known as the harmonic's order while the integer $\ell \geq 0$ is the degree of the harmonic. The harmonic basis functions are defined using the associated Legendre functions $P_{\ell}^{m}$ as

$$
Y_{\ell}^{m}= \begin{cases}\frac{2 \ell+1}{4 \pi} P_{\ell}^{0}(\cos (\phi)), & \text { if } m=0 \\ N(\ell, m) P_{\ell}^{m}(\cos (\phi)) \cos (m \theta), & \text { if } m>0 \\ N(\ell, m) P_{\ell}^{-m}(\cos (\phi)) \sin (m \theta), & \text { if } m<0\end{cases}
$$

where $N(\ell, m)=\sqrt{2} \sqrt{\frac{2 \ell+1}{4 \pi} \frac{(\ell-m) !}{(\ell+m) !}}$ is a normalization constant. Note that $\theta$ represents the azimuthal angle (from the positive $\mathrm{x}$-axis) and $\phi$ the polar angle (from the positive $\mathrm{z}$ axis) of the spherical coordinate system. For fiber or diffusion ODFs, only the even-numbered orders are required due to the antipodal symmetry of the diffusion MRI imaging sequence [9]. Although our method works for any $\ell$, typically degrees $\ell \leq 8$ are used as larger-degree harmonic representations often over-fit the dMRI data [8].

Considering $\psi_{v}(\theta, \phi)$ as our ODF from (2) in the spherical coordinate system, we can greatly simplify the edge weight integration. Using the linearity of integration, we obtain

$$
\int_{\beta} \psi_{v}(\theta, \phi) d S=\sum_{\ell=0}^{K} \sum_{m=-\ell}^{\ell} F_{\ell}^{m} \int_{\beta} Y_{\ell}^{m}(\theta, \phi) d S .
$$

Note that the effect of the dMRI data is limited to the setting of the constants $F_{\ell}^{m}$ and that the integration occurs only over the spherical harmonic basis functions. As a result, the integral in (5) does not vary over the graph and only needs to be calculated once for each edge direction. Given integral limits defining the solid angle $\beta$, the integration in (5) can be performed offline to speed up later graph constructions.

\section{INTEGRATION LIMITS}

While we have been able to reduce the number of integrals that need to be calculated, we have yet to show that the integrals in (5) can be calculated analytically. The first step in achieving this goal is to rewrite the integral limits in a way that facilitates an analytical solution.
In $[2,4]$, the area of integration includes all directions $(\theta, \phi)$ that are within an angular distance $\alpha$ of the edge direction (denoted as $\left.\left(\theta_{0}, \phi_{0}\right)\right)$. This results in a cone of influence with its central axis along $\left(\theta_{0}, \phi_{0}\right)$ and an apex angle of $2 \alpha$. The spherical cap created by this cone is our area of integration, $\beta$, and is usually expressed as a solid angle. The angle $\alpha$ is usually chosen to obtain a spherical cap with a solid angle of $\frac{4 \pi}{M}$, where $4 \pi \mathrm{sr}$ is the solid angle of the complete sphere and $M$ is the neighborhood size (e.g., $M=26$ for 26-connectivity).

In general, integrating over the spherical cap of this cone leads to complicated limits of integration that make an analytical solution to the integral difficult, if not impossible [10]. We have, however, identified a special case where these integral limits simplify into constant values. If an edge is aligned with the direction $\phi=0$ ( or $\phi=\pi$ ), then the small circle used to define our limits of integration can be traced by setting $\phi=\alpha$ and letting $\theta$ vary between zero and $2 \pi$. The spherical cap defining our area of integration becomes the set of concentric circles centered at $(0,0)$ and having angular radii less than $\alpha$. As such, we can integrate this spherical cap with the following integral limits:

$$
\phi \in[0, \alpha] \quad \text { and } \quad \theta \in[0,2 \pi] .
$$

These constant integral limits are imperative in obtaining an analytical solution to (2), as we will show in Section 4. Note that these integral limits are only valid for the special case where the edge is aligned with $\phi=0$. In order to be able to use these integral limits for any arbitrarily-directed edge, we must first rotate the spherical harmonic expansion so as to align the edge in question with $\phi=0$. The rotation of a spherical harmonic expansion is performed by multiplying the coefficients $F_{\ell}^{m}$ by a rotation matrix $R_{\ell}$. We create the rotation matrices using the recurrence relations defined in [11] to obtain rotated spherical harmonic coefficients $\mathcal{F}_{\ell}^{m}$.

While there is a computational expense in rotating the ODFs, we will show in the following two sections that this cost is not prohibitave. Further, we note that these rotation matrices can be precomputed and only need to be computed once for a given graph connectivity scheme.

\section{ANALYTICAL EDGE WEIGHT INTEGRATION}

At this point, we have shown that by using spherical harmonics and rotations, we can simplify (2) to

$P_{\text {odf }}\left(v, \vec{r}_{u v}\right)=\sum_{\ell=0}^{K} \sum_{m=-\ell}^{\ell} \mathcal{F}_{v, \ell}^{m} \int_{0}^{\alpha} \int_{0}^{2 \pi} Y_{\ell}^{m}(\theta, \phi) \sin (\phi) d \theta d \phi$

where $\mathcal{F}_{v, \ell}^{m}$ are the rotated spherical harmonic coefficients for voxel $v$. Note that $d S$ has been replaced with the appropriate substitution $d S=\sin (\phi) d \theta d \phi$. What remains to be shown 
is that the simplified integral in (7) can be solved analytically and that solving (7) analytically is more computationally efficient than performing numerical integration.

Given the real spherical harmonic basis and the integral limits from (6), we can prove that for $m \neq 0$ :

$$
\int_{0}^{\alpha} \int_{0}^{2 \pi} Y_{\ell}^{m}(\theta, \phi) \sin (\phi) d \theta d \phi=0 .
$$

This result can be seen by expanding (8). For $m>0$, we see that (8) can be rewritten as

$$
\begin{aligned}
& \int_{0}^{\alpha} \int_{0}^{2 \pi} N(\ell, m) P_{\ell}^{m}(\cos (\phi)) \cos (m \theta) \sin (\phi) d \theta d \phi \\
& =N(\ell, m) \int_{0}^{\alpha} P_{\ell}^{m}(\cos (\phi)) \sin (\phi) \int_{0}^{2 \pi} \cos (m \theta) d \theta d \phi \\
& =N(\ell, m) \int_{0}^{\alpha} P_{\ell}^{m}(\cos (\phi)) \sin (\phi)\left[\frac{\sin (m \theta)}{m}\right]_{0}^{2 \pi} d \phi=0
\end{aligned}
$$

as $\sin (2 \pi m)=\sin (0)$. The same can be shown for $m<0$.

This result is encouraging as it allows us to further reduce the number of integrals we need to compute in order to obtain the edge weight defined in (7), thereby increasing the computational efficiency of the integration. Further, we require only the rotated spherical harmonic coefficients $\mathcal{F}_{\ell}^{m}$ for cases where $m=0$. The other rotated spherical harmonic coefficients no longer need to be calculated.

Finally, for the spherical harmonics basis functions for $m=0$, it can be shown that

$$
\begin{aligned}
\int_{0}^{\alpha} \int_{0}^{2 \pi} Y_{\ell}^{0}(\theta, \phi) \sin (\phi) d \theta d \phi \\
\quad=-2 \pi \sqrt{\frac{2 \ell+1}{4 \pi}} \int_{1}^{\cos (\alpha)} P_{\ell}^{0}(z) d z
\end{aligned}
$$

where $z=\cos (\phi)$ and $d z=-\sin (\phi) d \phi$.

The result in (10) shows that the ODF edge weight integration reduces to integrations over the Legendre polynomials. Table 1 shows the first few Legendre polynomials used in our edge weight integration as well as their indefinite integrals (ignoring the additive constants). Higher order Legendre polynomials follow the same pattern as in Table 1 and are easily analytically integrable. Evaluating the integrals in Table 1 using the limits from (10) completes the integration in (7).

\begin{tabular}{c|c|c|c}
$\ell$ & 0 & 2 & 4 \\
\hline$P_{\ell}(z)$ & 1 & $\frac{1}{2}\left(3 z^{2}-1\right)$ & $\frac{1}{8}\left(35 z^{4}-30 z^{2}+3\right)$ \\
$\int P_{\ell}(z)$ & $\mathrm{z}$ & $\frac{1}{2}\left(z^{3}-z\right)$ & $\frac{1}{8}\left(7 z^{5}-10 z^{3}+3 z\right)$
\end{tabular}

Table 1. The Legendre polynomials and their indefinite integrals of even degree up to $\ell=4$.

\section{EXPERIMENTAL RESULTS}

We aim to show the gains in the numerical accuracy and computational efficiency of our analytical solution compared to commonly adopted numerical schemes. These gains will be shown both separately and in the context of an existing dMRI analysis algorithm. The following results were obtained using three 3D diffusion MRI volumes publicly available from the 2009 Pittsburgh Brain Competition [12]. Each data set was comprised of 256 diffusion weighted images acquired with a b-value of $1500 \mathrm{~s} / \mathrm{mm}^{2}$ at $2 \mathrm{~mm}$ isotropic voxel resolution. Fiber ODFs were computed as in $[8,9]$ with a spherical harmonic expansion of degree $\ell=6$. Numerical integration was performed using tessellations of an icosahedron.

\begin{tabular}{c|c|c|c|c}
\multirow{2}{*}{ Method } & \multicolumn{2}{c|}{ Comp. Time (s) } & \multicolumn{2}{c}{ NRMS Error (\%) } \\
& Mean & Std. Dev. & Mean & Std. Dev. \\
\hline \multicolumn{1}{c|}{ Proposed } & 3.90 & 0.16 & $\epsilon$ & $\epsilon$ \\
\hline $\begin{array}{c}1 \text { st } \\
\text { order }\end{array}$ & 22.59 & 0.57 & 11.98 & 0.17 \\
\begin{tabular}{c}
42 pts.) \\
\hline $\begin{array}{c}2 \mathrm{nd} \\
\text { order } \\
\text { (162 pts.) }\end{array}$
\end{tabular} & 41.57 & 1.78 & 4.73 & 0.02 \\
\hline $\begin{array}{c}\text { 3rd } \\
\text { order } \\
\text { (642 pts.) }\end{array}$ & 171.04 & 6.20 & 2.41 & 0.01 \\
\hline
\end{tabular}

Table 2. Computation time and normalized root-meansquared error results for various methods of calculating $P_{\text {odf }}$ in (2). Results shown for our exact method versus numerical integration using different order tesselations of an icosahedron. Note our proposed method gives accurate results within machine precision $\epsilon$.

Table 2 displays the mean and standard deviation of the normalized root mean squared (NRMS) error for all values of $P_{\text {odf }}$ calculated over the approximately 200,000 fiber ODFs in each volume for different order tessellations (i.e., numerical approximation with 42, 162, and 642 vertices respectively). Also shown is the mean and standard deviation in computation time for solving (2) for a dMRI volume. Timing results for our proposed solution include the spherical harmonic rotations mentioned in Section 3. As the quality of the tessellation improves, the numerical approximations approach our exact result. However, as the numerical approximation improves, so too does the computational intensity. Yet, our solution is able to compute (2) roughly 5.8 times faster than the coarsest numerical approximation and almost 44 times faster than numerical approximation using a third-order tessellation.

We applied our exact ODF integration to the graph-based tractography algorithm in [4]. Sample results are shown in Figure 1 for seeds regions in the body of the Corpus Callosum 


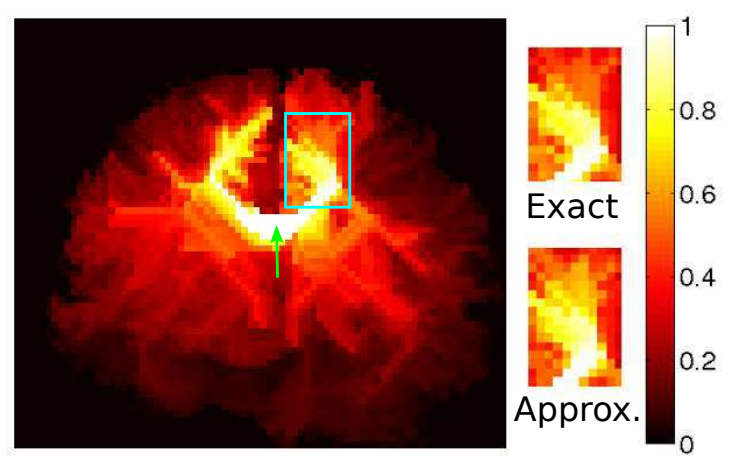

(a) Seed ROI in Corpus Callosum body (green arrow)

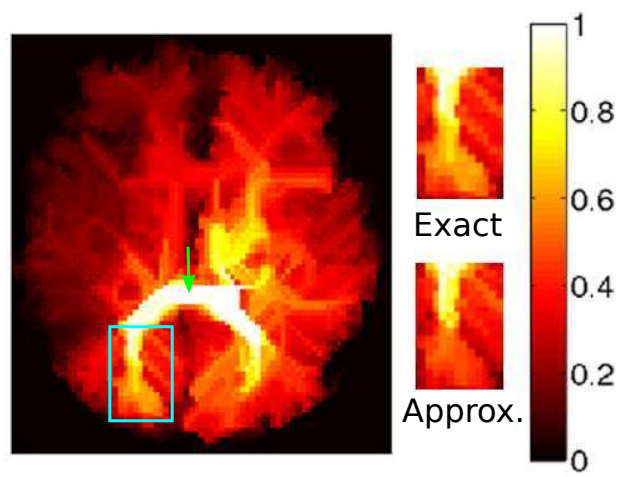

(b) Seed ROI in Splenium (green arrow)

Fig. 1. Connection probabilities using the exact and the numerical approximation of $P_{\text {odf }}$ in (2). The tractography results shown are based on our exact solution for obtaining the graph edge weights. The regions highlighted in cyan are compared to results for numerical approximation with $2^{\text {nd }}$ order tesselation of an icosahedron. Maximum intensity projections along the coronal and axial planes are shown in (a) and (b) respectively. Note the qualitative differences in connection probabilities in the highlighted regions.

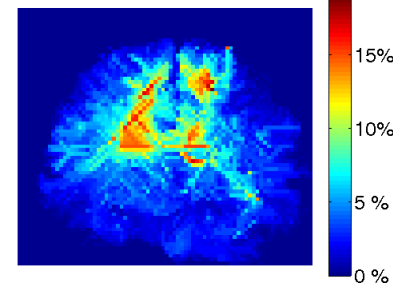

(a) Corpus Callosum

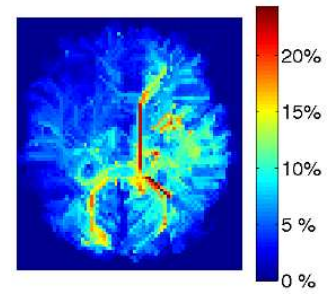

(b) Splenium
Fig. 2. Percentage error in connection probabilities for the examples in Figure 1. Maximum intensity projections along the coronal and axial planes are shown respectively. Note how the error from numerical approximation reaches over $15 \%$

(CC) and in the Splenium (shown by the green arrows). Our exact ODF integration generates regions of high fiber probability that are less diffuse and extend further from the seed region. These results, highlighted respectively in the two displayed regions of interest (denoted in cyan), are likely due to the avoidance of error accumulation when approximating the integral in (2). Figure 2 shows the percentage error between the tractograms generated using the exact and approximate ODF integration solutions. Note that we are able to reduce error in the resulting tractograms by over $15 \%$ in places.

\section{CONCLUSION}

We have shown herein that numerical ODF integration has an effect on the accuracy of graph-based dMRI analysis algorithms. We presented an exact solution to the edge weight ODF integral that is both computationally efficient and numerically accurate. Results show that using this analytical edge weight integration speeds up and improves the accuracy of existing graph-based dMRI analysis algorithms [2, 3, 4].

\section{REFERENCES}

[1] S.Z. Li, Markov Random Field Modeling in Image Analysis, Springer, third edition, 2009.

[2] Y. Iturria-Medina, E.J. Canales-Rodríguez, L. Melie-García, P.A. Valdés-Hernández, E. Martínez-Montes, Y. Alemán-Gómez, and J.M. Sánchez-Bornot, "Characterizing brain anatomical connections using diffusion weighted MRI and graph theory," NeuroImage, vol. 36, pp. 645-660, 2007.

[3] A. Zalesky, "DT-MRI fiber tracking: A shortest paths approach," IEEE Trans. Med. Imag., vol. 27, no. 10, pp. 1458-1571, 2008.

[4] S.N. Sotiropoulos, L. Bai, P.S. Morgan, C.S. Constantinescu, and C.R. Tench, "Brain tractography using Q-ball imaging and graph theory: Improved connectivities through fibre crossings via a model-based approach," NeuroImage, vol. 49, pp. 2444-2456, 2010.

[5] M.-C. Chiang, A. D. Klunder, K. McMahon, G. I. de Zubicaray, M. J. Wright, A. W. Toga, and P. M. Thompson, "Information-theoretic analysis of brain white matter fiber orientation distribution functions," in Proc. of Info. Proc. for Med. Imag., 2007, vol. 20, pp. 172-182.

[6] K. M. Hasan, P. J. Basser, D. L. Parker, and A. L. Alexander, "Analytical computation of the eigenvalues and eigenvectors in DT-MRI," $J$. Mag. Res., vol. 152, pp. 41-47, 2001.

[7] H. Zhang, P. A. Yushkevich, D. C. Alexander, and J. C. Gee, "Deformable registration of diffusion tensor MR images with explicit orientation optimization," Med. Imag. Anal., vol. 10, pp. 764-785, 2006.

[8] M. Descoteaux, R. Deriche, T. R. Knösche, and A. Anwander, "Deterministic and probabilistic tractography based on complex fibre orientation distributions," IEEE Trans. Med. Imag., vol. 28, no. 2, pp. 269-286, 2009.

[9] B. Jian and B. C. Vemuri, "A unified computational framework for deconvolution to reconstruct multiple fibers from diffusion weighted MRI," IEEE Trans. Med. Imag., vol. 26, no. 11, pp. 1464-1471, 2007.

[10] J. R. Wertz, Spacecraft Attitude Determination and Control, D. Reidel, 1980.

[11] J. Ivanic and K. Ruedenberg, "Rotation matrices for real spherical harmonics. direct determination by recursion," J. Phys. Chem., vol. 100, pp. 6342-6347, 1996.

[12] W. Schneider and SchLab, "Pittsburgh brain competition," http://pbc.lrdc.pitt.edu/, accessed April 2009. 